\title{
Phenotypes of trpl Mutants and Interactions between the Transient Receptor Potential (TRP) and TRP-Like Channels in Drosophila
}

\author{
Hung-Tat Leung, Chaoxian Geng, and William L. Pak \\ Department of Biological Sciences, Purdue University, West Lafayette, Indiana 47907-1392
}

\begin{abstract}
The trp and trpl genes are thought to encode two classes of light-activated ion channels in Drosophila. A previous report indicated that a null trp/ mutant does not display any mutant phenotype. This lack of detectable mutant phenotypes made it difficult to suggest functions for the transient receptor potentiallike (TRPL) channel in photoreceptor responses. Here, the properties of trpl photoreceptor responses were studied by using electroretinogram (ERG) and intracellular recording techniques in combination with light stimuli of relatively long durations. Distinct mutant phenotypes were detectable under these conditions. These consisted of a reduced sustained component, oscillations superimposed on the response, a poststimulus hyperpolarization, and altered adaptation properties to dim background light. Comparison of photoreceptor responses obtained from wild
\end{abstract}

type, trp, and trp/ showed that the responses obtained from the trp and trp/ null mutants did not sum up to that of the wild-type response. To explain the nonlinear summation at the peak of the response, Reuss et al. (1997) proposed that $\mathrm{Ca}^{2+}$ ions entering through the TRP channel modulate TRP and TRPL channel activities differentially. However, nonlinear summation was present not only at the peak but throughout the duration of response. Two lines of evidence are presented to suggest that, in addition to the interaction proposed by Reuss et al. (1997), there are other forms of interactions between TRP and TRPL channels, probably involving the channel proteins themselves.

Key words: Drosophila; phototransduction; trpl phenotypes; TRP channel; TRPL channel; channel interactions
Drosophila photoreceptors respond to light with a depolarization mediated by a phospholipase C (PLC)-dependent signaling pathway (Bloomquist et al., 1988), resulting in the opening of two types of cation channels, transient receptor potential (TRP) and TRPlike (TRPL). The mechanism of activation of either channel is not known. The TRP and TRPL channel subunits are encoded by the transient receptor potential (trp) and trp-like ( $t r p l)$ genes, respectively (Montell and Rubin, 1989; Wong et al., 1989; Phillips et al., 1992). The protein products of trp and trpl share 39\% amino acid identity and some homology to neuronal voltage-gated channel subunits, although neither TRP nor TRPL is voltage-gated (Stühmer et al., 1989; Phillips et al., 1992). The TRP channel is highly calcium-permeable, whereas the TRPL channel is nonspecifically cation-selective (Hardie and Minke, 1992; Niemeyer et al., 1996; Reuss et al., 1997).

Previous workers have demonstrated that trp mutants exhibit severely impaired photoreceptor responses (Cosens and Manning, 1969; Minke et al., 1975; Pak, 1979; Minke, 1982). However, a null trpl mutant, trpl $^{302}$, has been reported not to show any mutant phenotype (Niemeyer et al., 1996), raising questions about the role of the TRPL channel in phototransduction. It was suggested that TRP and TRPL might play overlapping roles. Subsequently, Reuss et al. (1997) showed differences in ionic permeabilities between wild type and $\operatorname{trpl}^{302}$. However, the differences were subtle, and the question still remained whether $t r p l^{302}$ has a clear-cut phenotype. Previous studies of photoreceptor responses of $\mathrm{trpl}^{302}$ were performed on dissociated photoreceptors (Hardie, 1991), using patchclamp techniques (Hamill et al., 1981) and short-duration light stimuli. In an attempt to keep the preparations as physiologically intact as possible, we used living flies to perform extracellular

\footnotetext{
Received April 24, 2000; revised June 23, 2000; accepted July 6, 2000.

This work was supported by a grant from the National Eye Institute, National Institutes of Health (W.L.P.). The departmental shared confocal microscope facility was supported by National Science Foundation Grant BIR-9512962. We thank Dr. John A. Pollock of Carnegie Mellon University for providing anti-TRP antibodies and Dr. Charles Zuker of University California San Diego for making the $\operatorname{trpl} l^{302}$ mutant available.

Correspondence should be addressed to Dr. William L. Pak, Purdue University, Department of Biological Sciences, 1392 Lilly Hall, West Lafayette, IN 47907-1392. E-mail:wpak@bilbo.bio.purdue.edu.

Copyright (C) 2000 Society for Neuroscience $0270-6474 / 00 / 206797-07 \$ 15.00 / 0$
}

(electroretinograms, ERG) and intracellular recordings of photoreceptor responses. Moreover, we used light stimuli of relatively long durations to explore properties of the sustained component of the receptor potential. We show that, under these recording conditions, $\operatorname{trpl}^{302}$ photoreceptor responses do display distinct mutant phenotypes.

TRP and TRPL channel activities appear to influence each other. For example, the similarity in the current amplitudes obtained from $\mathrm{trpl}^{302}$ and wild type was explained in terms of differential regulation of the two channels by $\mathrm{Ca}^{2+}$ entering through the TRP channel (Reuss et al., 1997). These authors found that an increase in the internal $\mathrm{Ca}^{2+}$ concentration first facilitated and then suppressed the TRP channel while suppressing the TRPL channel. Thus, during the initial part of the photoreceptor response in wild-type flies the calcium ions coming in through the TRP channels would facilitate the activities of TRP channels but suppress those of TRPL channels. Because the TRP channels are the only major contributors to the early part of the response in both wild type and $\operatorname{trpl}^{302}$, their peak response amplitudes would be similar. Such interactions are important because they modulate and shape the responses of photoreceptors. We, therefore, sought to determine what other forms of interactions might be present between TRP and TRPL channels.

\section{MATERIALS AND METHODS}

Materials. The wild-type strain used was Oregon R. All flies were marked with the mutations $w$ (white) or cn bw (cinnabar brown) to remove the screening pigments in the eye. The $\operatorname{trpl}^{302}$ mutant was generated by Niemeyer et al. (1996). All other mutants were generated on an Oregon R background by ethylmethane sulfonate mutagenesis in this laboratory. The light stimuli, originating from a tungsten halogen lamp (Bausch \& Lomb, Rochester, NY), were delivered to the preparation with a fiber optics light guide. The unattenuated intensity at the level of the fly was $\sim 800 \mu \mathrm{W} / \mathrm{cm}^{2}$.

Electroretinograms (ERGs). The ERGs were obtained as described in Larrivee et al. (1981), using glass microelectrodes filled with Hoyle's saline. White light stimuli attenuated by two log units were used. Signals were filtered below $100 \mathrm{~Hz}$ and sampled at $5 \mathrm{kHz}$ with an analog-to-digital converter (Digidata 1200A), and the data were acquired and analyzed in a computer with Axoscope (Axon Instruments, Foster City, CA).

Intracellular recordings. Intracellular recordings were performed as described in Johnson and Pak (1986) on 1-d-old flies. The electrodes were introduced through a cut that covered small parts of both the cornea and the head. Vacuum grease was applied on the cut to prevent fluid loss. The 
recording electrodes had 30-50 $\mathrm{M} \Omega$ resistance when filled with $2 \mathrm{M} \mathrm{KCl}$. Signals were filtered out below $50 \mathrm{~Hz}$ and sampled at $2 \mathrm{kHz}$ with an analog-to-digital converter (Digidata 1200A), and the data were acquired and analyzed in a computer with Axoscope (Axon Instruments).

Western blot analysis. Flies were raised at $25^{\circ} \mathrm{C}$ in a $12 \mathrm{hr}$ light/dark illumination cycle. Heads were collected from six female and six male flies at $1 \mathrm{~d}$ after eclosion and homogenized in $60 \mu \mathrm{l}$ of SDS-PAGE sample buffer containing $50 \mathrm{~mm}$ dithiothreitol (DTT). The homogenate was boiled for $5 \mathrm{~min}$ and centrifuged $(12,000 \times g$ for $3 \mathrm{~min})$, and $10 \mu \mathrm{l}$ of the supernatant was loaded onto SDS/8\% acrylamide gels. To confirm the uniformity of total protein loading in each lane, we stained one of the several identically loaded gels with Coomassie blue. Western blots were generated by a standard protocol. The anti-TRP monoclonal antibody (Pollock et al., 1995) was used at 1:3000 dilution.

Confocal microscopy. After dissection, fly eyes were fixed in $4 \%$ formaldehyde (in PBS with $0.3 \%$ Triton X-100) for $1 \mathrm{hr}$ and incubated in PBS containing $4 \%$ normal goat serum for $2 \mathrm{hr}$. Filamentous actin of the rhabdomeres was stained with phalloidin-tetramethylrhodamine B isothiocyanate (Sigma, St. Louis, MO). Optical sections of $\sim 1 \mu \mathrm{m}$ thickness were viewed by confocal microscopy.

\section{RESULTS}

\section{Phenotypes of $\operatorname{trpl}^{302}$}

Among the parameters of photoreceptor responses that were examined by using ERG and intracellular recording techniques were (1) the time course of decay of the response during stimulus; (2) $V-\log I$ relations, which relate response amplitudes with the log of stimulus intensity, determined at two time points: the peak of the response and $2 \mathrm{sec}$ after the onset of response; (3) oscillations superimposed on the response; (4) poststimulus hyperpolarizations; (5) adaptation to background illuminations of different intensities; (6) speed of recovery from a previous stimulation (refractory period); and (7) response latency.

As reported by others (Niemeyer et al., 1996; Reuss et al., 1997), the peak response amplitudes obtained from $\operatorname{trpl}^{302}$ and wild type were similar (Fig. 1A,B). However, the sustained components of the two responses had very different amplitudes and time courses of decay. During a bright $20 \mathrm{sec}$ light stimulus the responses of both wild type and $\operatorname{trpl}^{302}$ declined from the peak amplitude to a lower sustained level (Fig. 1A). However, the $\operatorname{trpl}^{302}$ response declined to a much lower level so that the response remaining $20 \mathrm{sec}$ after the onset was significantly smaller in $\operatorname{trpl}^{302}$ than in wild type. The smaller sustained amplitudes of the trpl responses could be detected even $2 \mathrm{sec}$ after the stimulus onset. As before, the peak amplitudes of the wild-type and $\operatorname{trpl}^{302}$ responses were similar, but the amplitudes measured at $2 \mathrm{sec}$ after the onset of response were significantly smaller in $\operatorname{trpl}^{302}$ than in wild type (Fig. 1B). Tables 1 and 2 summarize the results of amplitude measurements at the response peak and $2 \mathrm{sec}$ after the response onset obtained at several stimulus intensities. Although the peak amplitudes were similar between wild type and $\operatorname{trpl}^{302}$ (Table 1), the normalized amplitudes measured at $2 \mathrm{sec}$ after the response onset were all significantly smaller in $\mathrm{trpl}^{302}$ than in wild type (Table 2).

In addition to the smaller sustained component, the $\operatorname{trpl}^{302}$ response often was accompanied by oscillations superimposed on the response during stimulus and a hyperpolarization immediately after the stimulus termination (Fig. $1 B-b$ ). To determine the frequency of occurrence of these two response phenotypes, we obtained ERGs from $30 \mathrm{trpl}^{302}$ and 30 wild-type flies. As summarized in Table 3, 80\% (24 of 30) and 37\% (11 of 30) of trpl ${ }^{302}$ flies exhibited oscillations and poststimulus hyperpolarizations, respectively, whereas none of the wild-type flies showed either property. Response oscillations and poststimulus hyperpolarizations also were examined in intracellularly recorded responses, allowing the average amplitude of hyperpolarizations to be determined. Of the 12 cells that were analyzed, 10 showed oscillations $(83 \%)$ and seven $(58 \%)$ showed hyperpolarizations, yielding similar frequencies of occurrence of these properties as in ERG recordings. The average amplitude of hyperpolarization in the seven cells was $2.2 \pm 0.6 \mathrm{mV}$ when light intensities were attenuated by two $\log$ units, i.e., $\log I / I_{0}$ $=-2$, where $I$ and $I_{0}$ are as defined in the Figure 1 legend.

Still another characteristic of $\operatorname{trpl}^{302}$ was its inability to adapt to dim background illuminations (Fig. 2). Light adaptation refers to

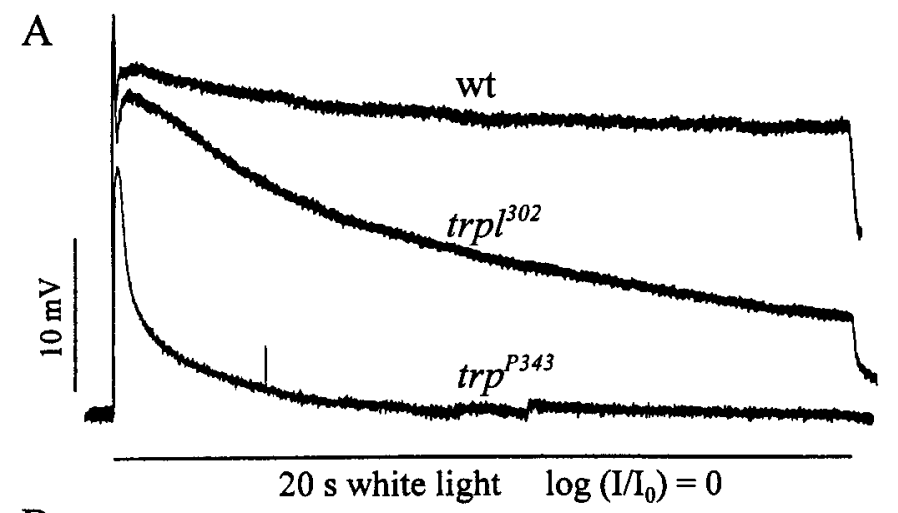

B
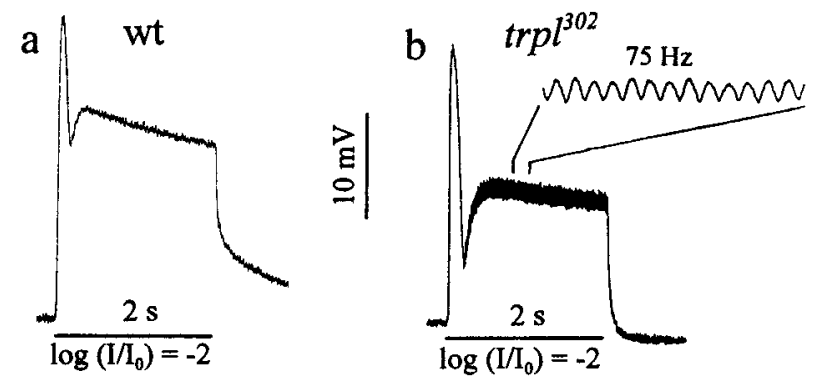

Figure 1. Electrophysiologically detectable phenotypes of $\operatorname{trpl}^{302}$. A, Comparison of representative receptor potentials elicited from wild type, $\operatorname{trpl}^{302}$ and $\operatorname{trp}^{P 343}$ by using prolonged stimuli. The $\operatorname{trpl} l^{302}$ receptor potential had a peak amplitude similar to that of wild type but a substantially smaller sustained component, although not as small as that of $\operatorname{trp}^{P 343}$. White light stimuli of $20 \mathrm{sec}$ duration were used without any attenuation $\left(\log I / I_{0}=0\right.$, where $I=$ stimulus intensity used and $I_{0}=$ maximum stimulus intensity available). $B$, Comparison of representative receptor potentials elicited from wild type $(a)$ and $\operatorname{trpl}^{302}(b)$ by using shorter stimuli viewed at faster sweep speed than in $A$. The stimuli were $2 \mathrm{sec}$ white lights attenuated by two $\log$ units $\left(\log I / I_{0}=-2\right)$. The trpl $^{302}$ receptor potential showed oscillations superimposed on the response, a reduced sustained amplitude, and a poststimulus hyperpolarization.

the ability of photoreceptors to adjust their sensitivity in response to changing background illuminations, allowing them to operate over a wide range of illuminations. Figure $2 A$ compares the $V$-log $I$ curves obtained from wild type and $\operatorname{trpl}^{302}$ normalized to the maximal response obtained from each cell, using the brightest stimulus available in the absence of background illumination. $V-\log$ $I$ curves obtained from wild-type photoreceptors shifted to increasingly higher intensities and saturated at progressively lower amplitudes as background illuminations increased in intensity (Fig. 2A). For a test stimulus of given intensity the largest response was obtained with no background illumination, and progressively smaller responses were obtained with background illuminations of increasing intensity (Fig. $2 A-a, B-a$ ). The trpl ${ }^{302}$ photoreceptors responded similarly at higher background illuminations, but the $V-\log I$ curve obtained at the lowest background that was tested was indistinguishable from that generated in the absence of background illumination [compare open diamonds $(-5 B G)$ with open squares (Dark $B G$ ) in Figure $2 A-b, B-b]$, suggesting that trpl photoreceptors failed to adapted to the dim background light.

Two other response properties that were tested were unaltered in $\operatorname{trpl}^{302}$, as was the case of peak amplitudes that were described previously. They were the refractory period and response latency. The refractory period was defined, in terms of the two-stimulus protocol illustrated in Figure 3, as the time required for the response to the second stimulus, $\mathrm{R} 2$, to attain nearly the same amplitude as the response to the first stimulus, R1. The refractory periods of wild-type and $\mathrm{trpl}^{302}$ responses were indistinguishable in this protocol. In both cases R2 had an amplitude similar to that of R1 when two strong $2 \mathrm{sec}$ stimuli, S1 and S2, were presented only $2 \mathrm{sec}$ apart. The response latency was defined as the time interval 
Table 1. Comparison of response amplitudes obtained from wild type and trpl $^{302}$

\begin{tabular}{lllllll}
$\begin{array}{l}\text { Light intensity } \\
\log I / I_{0}=\end{array}$ & 0 & -1 & -2 & -3 & \multicolumn{1}{c}{-4} & \multicolumn{1}{c}{-5} \\
\hline Wild type & $27.1 \pm 4.1$ & $26.0 \pm 5.9$ & $24.6 \pm 5.8$ & $18.8 \pm 5.1$ & $11.5 \pm 3.5$ & $4.4 \pm 1.6$ \\
trpl $^{302}$ & $27.0 \pm 3.6$ & $24.1 \pm 2.8$ & $21.4 \pm 3.2$ & $15.2 \pm 3.5$ & $8.7 \pm 2.6$ & $1.2 \pm 1.8$
\end{tabular}

Peak amplitude $(\mathrm{mV})(n \geqq 11)$.

\begin{tabular}{|c|c|c|c|c|c|}
\hline $\begin{array}{l}\text { Light intensity } \\
\log I / I_{0}=\end{array}$ & 0 & -1 & -2 & -3 & -4 \\
\hline Wild type & $70 \pm 7$ & $79 \pm 9$ & $74 \pm 10$ & $78 \pm 11$ & $83 \pm 11$ \\
\hline $\operatorname{trpl} l^{302}$ & $42 \pm 10$ & $53 \pm 11$ & $48 \pm 14$ & $48 \pm 13$ & $49 \pm 14$ \\
\hline
\end{tabular}

$(n \geqq 11)$.

\begin{tabular}{|c|c|c|c|c|c|}
\hline $\begin{array}{l}\text { Number of ERGs } \\
\text { showing }\end{array}$ & $\begin{array}{l}\text { Oscillations } \\
\text { alone }\end{array}$ & $\begin{array}{l}\text { Hyperpolarization } \\
\text { alone }\end{array}$ & Both & Neither & Total \\
\hline Wild type & 0 & 0 & 0 & 30 & 30 \\
\hline $\operatorname{trpl}^{302}$ & 14 & 1 & 10 & 5 & 30 \\
\hline
\end{tabular}

between the beginning of a stimulus and the onset of response. The latency of R2 was shorter than that of R1 in both $\operatorname{trpl}^{302}$ and wild-type flies. Moreover, the magnitudes of latency were also very similar (Fig. 3C).

\section{Phenotypes of trp}

The phenotypes of trp have been described extensively by previous workers (Cosens and Manning, 1969; Minke et al., 1975; Pak, 1979; Minke, 1982). The present study of trp was performed (1) to serve as controls for some of the experiments to be described and (2) to assess the relative contributions of TRP and TRPL activities to the photoreceptor response. As has been shown by others, a functionally null trp mutant, $\operatorname{trp}{ }^{P 34}$, had no sustained response during a strong prolonged light stimulation (Fig. $3 D$ ), a reduced peak amplitude (Fig. $3 A-c, D$ ), a longer-than-normal refractory period (Fig. $3 A-c$ ), and a longer-than-normal response latency (Fig. $3 B-c, C$ ). A near-null trp mutant, $\operatorname{trp}{ }^{P 301}$, gave similar results (see Fig. $5 A$, $B-b, C-b, D)$. As shown in Figure $3 D$, the peak amplitude of $\operatorname{trp}^{P 343}$, elicited by a bright stimulus, was $\sim 3 / 5$ that of the wild type, whereas that of $\operatorname{trpl}^{302}$ was approximately the same as wild type. There was no sustained component in a $\operatorname{trp}^{P 343}$ response, and there was a significantly reduced sustained component in $t_{r p l} l^{302}$. The summed response of $\operatorname{trp}^{P 343}$ and $t r p l^{302}$ had a peak amplitude larger than that of wild type and a sustained component smaller than that of wild type (Fig. 3D). This observation suggested that there might be functional interactions between TRP and TRPL channel activities. We therefore performed the following experiments to see whether there is any other evidence of possible interactions between TRP and TRPL and to obtain clues to the nature of the interactions, if any are present.

\section{TRPL helps to maintain a higher amount of TRP in Ina $D^{P 215}$}

Western blot analyses performed on 1-d-old $\operatorname{trpl}^{302}$ showed that the $\operatorname{trpl}^{302}$ mutation did not affect the amount of the TRP protein (Fig. $4 A$ ). The InaD gene encodes a PDZ protein to which several proteins involved in phototransduction, including TRP, bind to form a signaling complex (for review, see Montell, 1999; Tsunoda et al., 1999). In the InaD ${ }^{P 215}$ mutant the TRP protein reportedly cannot bind to the signaling complex because of a defect in the TRP-binding site on the INAD protein (Shieh and Zhu, 1996;
Chevesich et al., 1997; Tsunoda et al., 1997). Tsunoda et al. (1997) have reported that the amount of TRP was normal in newly eclosed InaD $D^{P 215}$ flies but decreased to an undetectable level by $10 \mathrm{~d}$ after eclosion. Consistent with this report, the amount of TRP in 1-d-old InaD $D^{P 215}$ fly heads was $\sim 70 \%$ of the wild-type amount (Fig. $4 A$ ). If the TRPL protein were removed from InaD ${ }^{P 215}$ (i.e., in the trpl $^{302}$ InaD $D^{P 215}$ double mutant), however, the amount of TRP was reduced to $<10 \%$ of the wild-type amount. To see whether degeneration of the rhabdomeres could be the cause of this large reduction in the amount of TRP in the $\operatorname{trpl}^{302} \operatorname{InaD^{P215}}$ double mutant, we performed confocal microscopy on the 1-d-old double mutant. The results (Fig. 4B) showed that the rhabdomeres of the double mutant were intact at this age, excluding degeneration of the rhabdomeres as a primary cause of the reduction in TRP in very young $\operatorname{trpl}^{302}$ InaD $D^{P 215}$. The above data, thus, suggested that the amount of TRP in 1-d-old InaD $D^{P 215}$ fly heads depended strongly on the presence of TRPL.

\section{TRP that is not bound to INAD contributes significantly to photoreceptor response in the presence, but not in the absence, of TRPL}

To explore how the binding of TRP to the signaling complex through INAD might affect the photoreceptor responses, we compared the responses of InaD $D^{P 215}$ and $\operatorname{trp}^{P 301} \cdot \operatorname{trp}^{P 301}$ is a near-null mutant that has no immunodetectable TRP protein but has small yet detectable physiological activities of TRP when examined in the double mutant $\operatorname{trpl}^{302} ; \operatorname{trp}^{P 301}$. Thus, 1-d-old InaD ${ }^{P 215}$ heads contained $\sim 70 \%$ of the wild-type amount of TRP (Fig. $4 A$ ), which presumably was not bound to the signaling complex, whereas $\operatorname{trp}^{P 301}$ heads contained a small, undetectable amount of TRP that, however, was bound properly to the complex. If the TRP protein that was not bound to the signaling complex were nonfunctional, then one might expect the phenotype of InaD $D^{P 215}$ to be similar to that of $\operatorname{trp}^{P 301}$. The comparison between the photoreceptor responses of $\operatorname{InaD} \dot{D}^{P 215}$ and $\operatorname{trp}^{P 301}$ showed otherwise. The responses of InaD ${ }^{P 215}$ were more wild-type-like (less severe) in the peak amplitude (Fig. $5 A$ ), amount of decay during light illumination (Fig. 5A), refractory period (Fig. 5B), and response latency (Fig. $5 C, D)$ than those of $\operatorname{trp}^{P 301}$. The results suggested that either some TRP remained bound to the signaling complex in InaD ${ }^{P 215} \mathrm{mu}-$ 
A

Figure 2. Inability of $\operatorname{trpl}^{302}$ to adapt to a dim background illumination. $A, V-\log I$ curves for wild type $(a)$ and $\operatorname{trpl}^{302}(b)$ determined at four different intensities of background illumination. $V-\log I$ curves relate the response amplitudes to relative stimulus intensities, which are given in log units. Both the test and background stimuli were white lights. Before each test stimulus a background light of $1 \mathrm{~min}$ duration was turned on first, and the 2 sec test stimulus was presented at the very end of the 1 min background. For each cell that was examined, all responses were normalized with respect to the maximal peak amplitude obtained in that cell by using the brightest stimulus $\left(\log I / I_{0}=0\right)$ in the absence of background illumination $(n=5)$. The average maximal peak amplitudes obtained from wild type and $\mathrm{trpl}^{302}$ were $28 \pm 4.2 \mathrm{mV}(n=5)$ and $26.1 \pm 3.3 \mathrm{mV}(n=5)$, respectively. Dark $B G$, No background illumination (open squares); $-5 B G$, background illumination attenuated by five $\log$ units (open diamonds); $-4 B G$, attenuated by four log units (open circles); -3 $B G$, attenuated by three $\log$ units (open triangles). Unlike in wild type, the $V$-log $I$ curve of trpl $^{302}$ obtained at -5 BG is indistinguishable from that obtained in Dark BG. $B$, Receptor potentials obtained from wild type $(a)$ and $\operatorname{trpl}^{302}(b)$ by using maximum intensity white test stimuli at different background intensities and by using the protocol described in $A$. In $t r p l^{302}$ the receptor potentials recorded in dark and $-5 \log$ backgrounds are very similar in amplitude and waveform.

$\mathbf{B}$
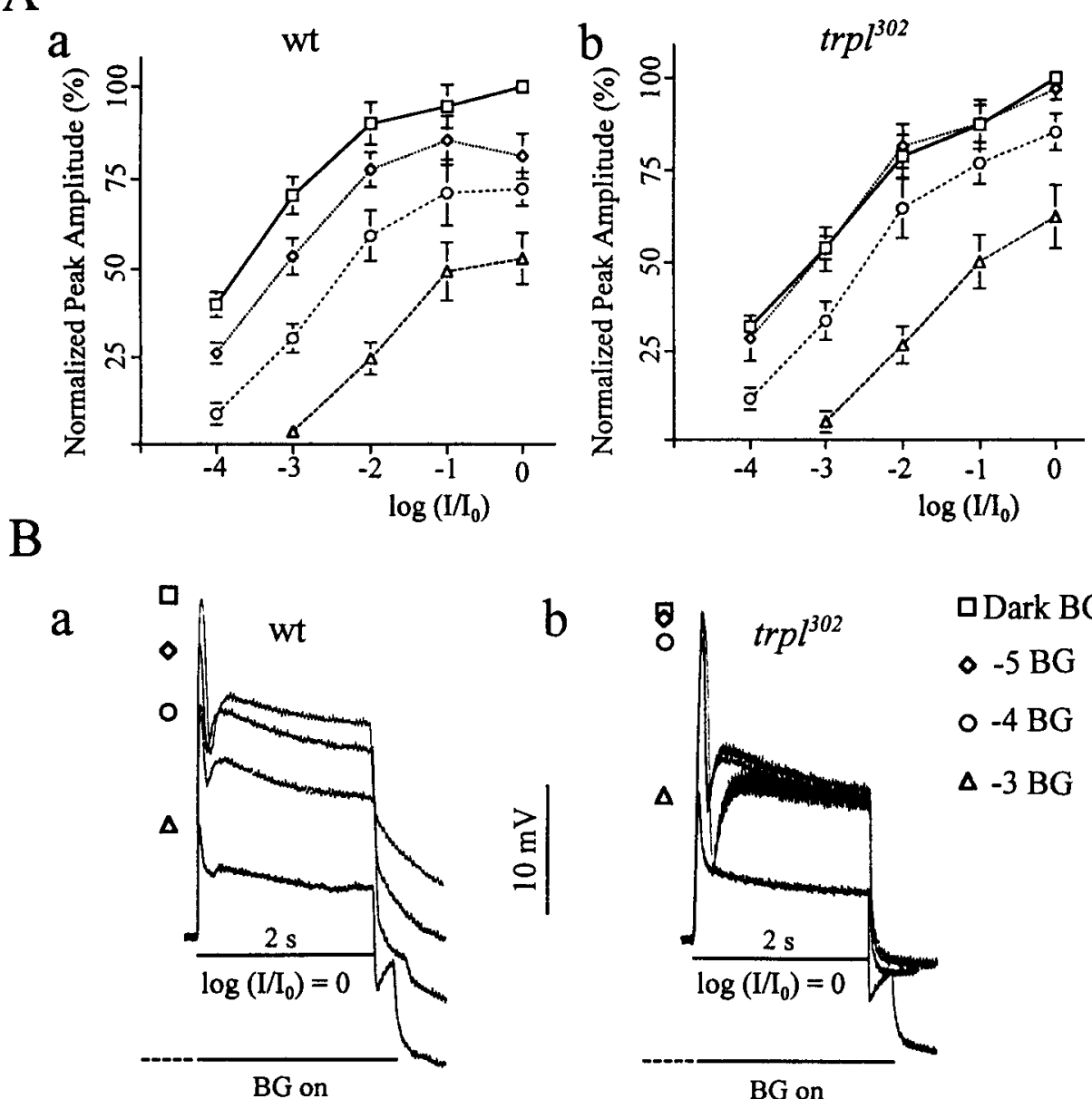

口Dark BG

$\diamond-5 \mathrm{BG}$

$0-4 \mathrm{BG}$

$\Delta-3 \mathrm{BG}$
Figure 3. Comparison of the refractory periods and response latencies of the receptor potentials obtained from wild type, $\operatorname{trpl}^{302}$, and $t r p^{P 343}$. The stimulus protocol is shown at the top. After a 2 min dark adaptation two $2 \mathrm{sec}$ stimuli ( $S 1$ and $S 2$ ) were presented $20 \mathrm{sec}$ apart, and the corresponding responses $(R 1$ and $R 2)$ were recorded. $A$, The responses, R1 and R2, obtained in the above protocol are shown superimposed to allow for a comparison of amplitudes: $a$, wild type; $b$, trpl $^{302}$; $c, \operatorname{trp}^{P 343}$. The term "refractory period" refers to the time required for the second response $(R 2)$ to attain a response amplitude similar to that of the first $(R 1)$. R2s of both wild type and $\operatorname{trpl}^{302}$ have amplitudes similar to those of R1s. R2 of $\operatorname{trp}^{P 343}$, however, is much smaller than that of R1. The stimulus intensity was attenuated by one $\log$ unit $\left(\log I / I_{0}=-1\right) . B$, The initial $120 \mathrm{msec}$ of the responses shown in $A$ are presented at a higher sweep speed than in $A$ to allow for a comparison of latencies: $a$, wild type; $b$, trpl $l^{302} ; c, \operatorname{trp}^{P 343}$ Arrows indicate the beginning of light stimuli. The response latency is defined as the time between the beginning of stimulus and the onset of the response. $C$. Histogram showing the response latencies of R1 and R2 obtained from wild type, $\operatorname{trpl}^{302}$, and $\operatorname{trp}^{P 343}(n=10)$. In both wild type and $\mathrm{trpl}^{302}$ the response latencies of $\mathrm{R} 1$ and $\mathrm{R} 2$ are similar in magnitude, and in both the R2 latency is significantly shorter than that of R1. In $\operatorname{trp}^{P 343}$, on the other hand, both R1 and R2 latencies are much longer than those of the other genotypes; moreover, the R2 latency is significantly longer than that of R1. D, Summing the trpl to2 $^{302}$ and $\operatorname{trp}^{P 343}$ responses does not reproduce the wild-type response. The shaded area represents the summation of the $\operatorname{trpl}^{302}$ and $\operatorname{trp}^{P 343}$ responses. The summed response has a larger peak amplitude but a smaller sustained component than the wild-type response.

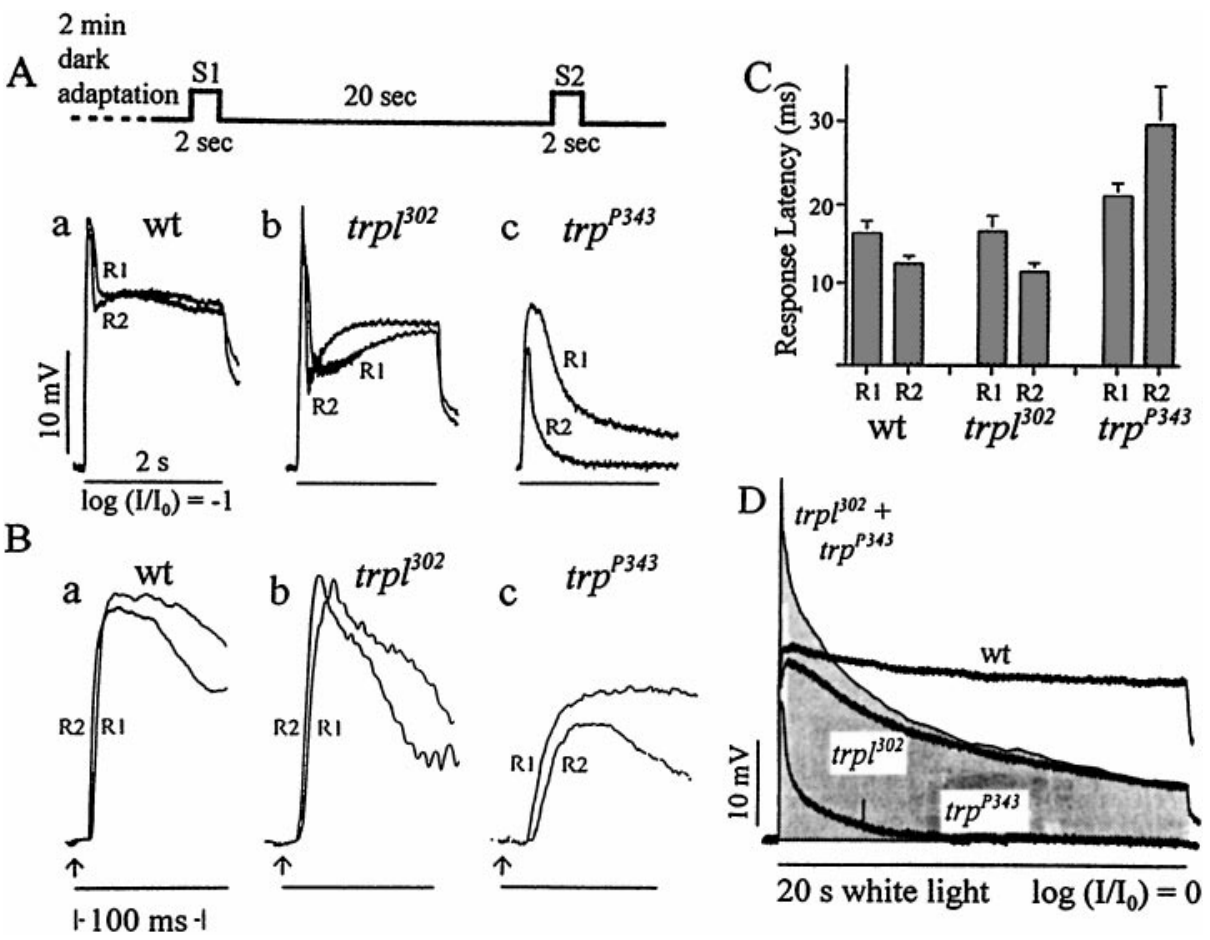

$\mathrm{D} \mid \begin{gathered}\operatorname{trpl}^{302}+ \\ \operatorname{trp}^{p 34}\end{gathered}$ 


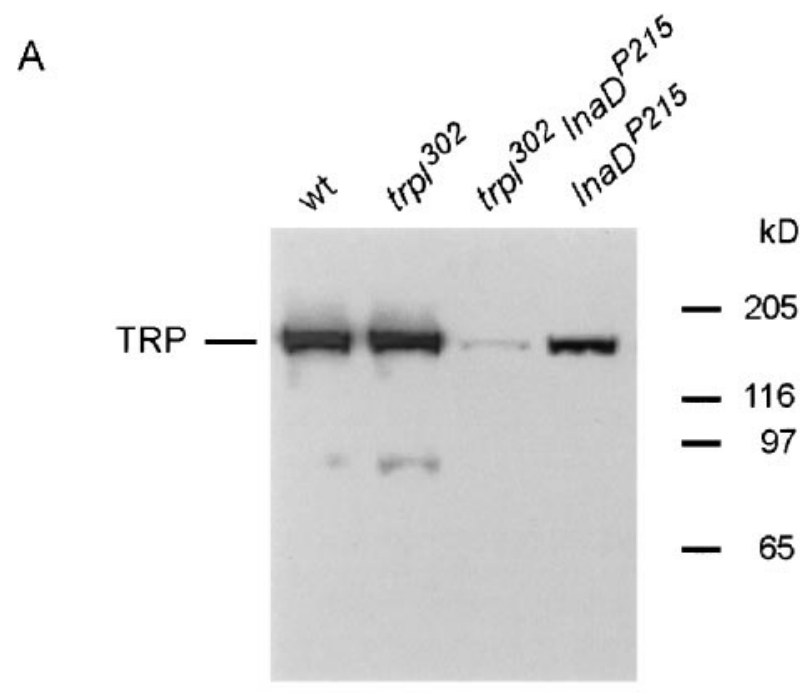

B

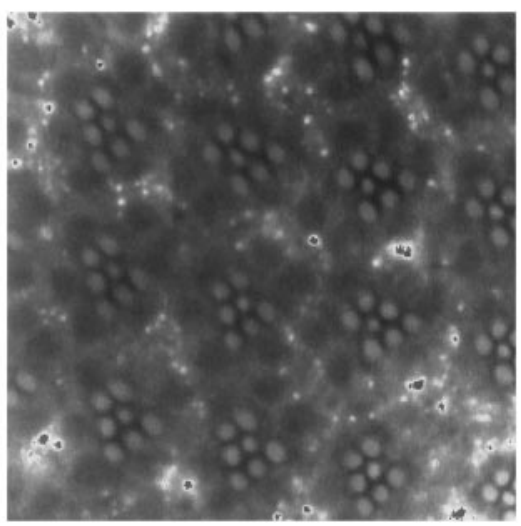

Figure 4. $A$, Western blot analysis showing the relative quantity of the TRP protein in wild type, $\operatorname{trpl}^{302}$, $\operatorname{trpl}^{302} \operatorname{Ina} D^{P 215}$, and InaD ${ }^{P 215}$. The amount of TRP in $\operatorname{trpl}^{302}$ is indistinguishable from that in wild type. Although the amount of TRP in InaD $D^{P 215}$ is reduced only slightly, that of trpl $^{382}$ InaD $D^{P 215}$ is $<10 \%$ of the wild-type amount. $B$, Confocal micrograph showing normal-looking rhabdomeres of the $\operatorname{trpl}^{302}$ InaD ${ }^{P 215}$ double mutant. The rhabdomeres were visualized by staining F-actin with phalloidin.

tants or the TRP channels that were not bound to the signaling complex contributed significantly to photoreceptor responses.

A very different picture emerged if the TRPL protein were removed from the above two mutants via the construction of the double mutants $\operatorname{trpl}^{302} \operatorname{InaD}^{P 215}$ and $\operatorname{trpl}^{302} ; \operatorname{trp}^{P 301}$. As may be seen in Figure $6 \mathrm{~A}$, the responses of both $\operatorname{trpl}^{302} \operatorname{InaD}^{P 215}$ and $\operatorname{trpl}^{302}$; $\operatorname{trp}{ }^{P 301}$ were small and transient, but the response amplitude of trpl $^{302}$ InaD ${ }^{P 215}$ was significantly smaller than that of $\operatorname{trpl}^{302} ; \operatorname{trp}^{P 301}$. The comparison of such other properties as the refractory period (Fig. 6B) and response latency (Fig. 6C,D) further showed that these phenotypes of $\operatorname{trpl}^{302}$ InaD ${ }^{P 215}$ were also more severe than those of the $\operatorname{trpl}^{302}$;rp ${ }^{P 301}$. Thus, in the presence of TRPL, TRP that was not bound to INAD contributed significantly to photoreceptor responses (InaD ${ }^{P 215}$ vs $\operatorname{trp}^{P 301}$ ), but in the absence of TRPL, TRP that was not bound to INAD was unable to contribute significantly to responses (trpl ${ }^{302}, \operatorname{trp}^{P 301}$ vs $\operatorname{trpl}^{302} \operatorname{InaD}^{P 215}$ ). Although the amount of detectable TRP in $\operatorname{trpl}^{302}$ InaD $D^{P 215}$ was much lower than in wild type (see Fig. $4 A$ ), this reduced TRP content could not account for its phenotypes, because the phenotypes were much more severe than those of $\operatorname{trpl}^{302}, \operatorname{trp}^{P 301}$, which had no detectable amount of TRP. The fact that the ability of non-INADbound TRP to contribute to photoreceptor responses depends on the presence of TRPL suggests that there may be interactions between TRPL and the non-INAD-bound TRP protein.

\section{DISCUSSION}

We have shown that the $\operatorname{trpl}^{302}$ mutants have clearly detectable mutant phenotypes. The absence of phenotype reported by previous workers may be attributable to differences in recording procedures. Previous studies of the $\operatorname{trpl}^{302}$ mutants were done by using the whole-cell patch-clamp technique on dissociated photoreceptors, whereas the present studies were performed by using the ERG and intracellular recording techniques on photoreceptors of intact, living flies. Perhaps an even more important difference might be the duration of stimuli used to elicit photoreceptor responses. Previous workers used light pulses of millisecond durations, whereas we used stimulus durations of seconds to tens of seconds. Properties of photoreceptor responses that are readily detectable with short-duration light flashes, such as the peak amplitude and response latency, indeed are essentially indistinguishable between $\operatorname{trpl}^{302}$ and wild type. However, a number of readily detectable phenotypes become apparent if longer stimulus durations are used and recordings are made from living flies. These include oscillations, poststimulus hyperpolarizations, reduced sustained components, and altered adaptation properties. These results suggest that the TRPL channels may have a role in sustaining the photoreceptor response during prolonged illuminations and in adaptation to dim light stimuli, because eliminating the TRPL channels (i.e., in trpl $^{302}$ ) substantially reduces the sustained component (see Fig. $1 A$ ) and makes the photoreceptors insensitive to dim background illuminations (see Fig. 2). However, complicating the interpretation regarding its role in sustaining the response is the observation that, in the absence of TRP channels (i.e., in $\operatorname{trp}^{P 343}$ ), the TRPL channels essentially make no contributions to the sustained component (see Fig. 1A). It thus may be that both classes of channels contribute to the sustained component and that they both must be present to allow mutual interactions so that normal channel outputs might be generated.

The summed amplitude of $\operatorname{trp}^{P 343}$ and $\operatorname{trpl} l^{302}$ mutant receptor potentials is larger at the peak but smaller throughout the sustained part of the response than the wild-type response amplitude (see Fig. 3D). As discussed previously, Reuss et al. (1997) proposed that the nonlinear summation at the peak of the response arises because $\mathrm{Ca}^{2+}$ ions entering through the TRP channel suppress the TRPL channel activity. Thus, in this form of TRP-TRPL interaction the current entering through one class of channel influences the activities of both the same and the other class of channels. For the purpose of present discussion, this form of interactions may be referred to as "functional interactions." However, nonlinear summation of the two channel activities is present not just at the peak but throughout the response. Moreover, the difference between the summed mutant amplitude and the wild-type amplitude is positive at the peak of the response but reverses in sign to be negative throughout the sustained component (see Fig. $3 D$ ). It is unlikely that a single mechanism, e.g., that proposed by Reuss et al. (1997), is responsible for the observed nonlinear summation of mutant responses throughout their entire time course.

The idea that other forms of interactions may be present in addition to "functional interactions" is supported by the results obtained with InaD $D^{P 215}$ and $t r p l^{302}$ InaD ${ }^{P 215}$ mutants. The amount of TRP in 1-d-old InaD $D^{P 215}$ mutants is substantially larger than that in the $\operatorname{trpl}^{302}$ InaD $D^{P 215}$ double mutants of the same age (see Fig. 4A). Moreover, the photoreceptor potential recorded from InaD $D^{P 215}$ is large and robust (see Fig. $5 A$ ), whereas that recorded from the double mutant $\operatorname{trpl}^{302}$ InaD ${ }^{P 215}$ is very small-even smaller than that of $\operatorname{trpl}^{302}$;rp ${ }^{P 301}$ (see Fig. 6A), which has no immunodetectable TRP protein. Several groups have shown previously, via in vitro binding assays, that the TRP protein is unable to bind to the INAD protein in InaD ${ }^{P 215}$ mutants (Shieh and Z hu, 1996; Chevesich et al., 1997; Tsunoda et al., 1997). The above results thus suggest that the presence of the TRPL protein somehow stabilizes the TRP protein even when the latter is not bound to the INAD protein and allows TRP to contribute substantially to the photoreceptor response. If one supposes that the TRPL protein is associated with the signaling complex, as suggested by Xu et al. (1998), a 
Figure 5. Comparison of the properties of the receptor potentials obtained from InaD ${ }^{P 215}$ and $\operatorname{trp}^{P 301}$. A, Representative receptor potentials recorded from wild type, InaD ${ }^{P 215}$, and $t r p^{P 301}$ by using prolonged, unattenuated white light stimuli. Although both InaD ${ }^{P 215}$ and $\operatorname{trp}^{P 301}$ receptor potentials decay toward the baseline, the InaD ${ }^{P 215}$ receptor potential decays more slowly than that of $\operatorname{trp}^{P 301} . B-D$, The differences in the properties of $\operatorname{In} a D^{P 215}$ and $\operatorname{trp}^{P 301}$ responses revealed in the two-stimuli protocol described in Figure 3. As in Figure 3, $A$ and $B$, the R1 and R2 responses are shown superimposed in $B$ and $C$. In $B$, the R2 amplitude is a significantly larger fraction of the R1 amplitude in InaD ${ }^{P 215}$ than in $\operatorname{trp}{ }^{P 301}$ at any point in the response time course so that the time integral of R2 is a significantly larger fraction of that of $\mathrm{R} 1$. $C$, The initial $130 \mathrm{msec}$ portions of the responses of InaD ${ }^{P 215}$ and $\operatorname{trp}^{P 301}$ in $B$ are presented at a higher sweep speed to allow for a comparison of latencies. $D$, Histogram comparing the response latencies of $\mathrm{R} 1$ and $\mathrm{R} 2$ from analysis of records similar to those in $C$ $(n=10)$ obtained from wild type, InaD ${ }^{P 215}$, and $\operatorname{trp}{ }^{P 301}$. The response latency of $\mathrm{R} 2$ is shorter than that of R1 in wild type, approximately the same as that of R1 in InaD ${ }^{P 215}$, and longer than that of R1 in $\operatorname{trp}^{P 301}$.
Figure 6. The effects of the $\mathrm{trpl}^{302}$ mutation on trp ${ }^{P 301}$ and InaD $D^{P 215}$ mutants. $A$, Comparison of representative receptor potentials recorded from wild type and the double mutants trpl $^{302}$ InaD $D^{P 215}$ and trpl $^{302}$, trp $^{P 301}$, using $2 \mathrm{sec}$ white light stimuli. The receptor potentials elicited from both trpl $^{302} I n a D^{P 215}$ and trpl $^{302} ; \operatorname{trp}^{P 301}$ are small and transient, but the response amplitude of $\operatorname{trpl}^{302} ; \operatorname{trp}^{P 301}$ is significantly larger than that of trpl $^{302}$ InaD $D^{\text {P215 }}$ $B, C$, Properties of responses revealed in twostimuli protocol (see Fig. 3). $B$, Comparison of R1 and R2 amplitude and waveform. R2 is a significantly larger fraction of R1 in $\mathrm{trpl}^{302}$; $\operatorname{trp}^{P 301}$ than in $\operatorname{rrpl}^{302}$ InaD ${ }^{P 215}$. C , The initial $120 \mathrm{msec}$ portions of the responses of trpl $^{302}$ InaD ${ }^{P 215}$ and trpl ${ }^{302}$; trp $^{P 301}$ in $B$ are presented at a higher sweep speed to allow for a comparison of latencies. $D$, Histogram comparing the response latencies of $\mathrm{R} 1$ and $\mathrm{R} 2$ obtained from wild type, $\operatorname{trpl} l^{302}, \operatorname{trp} p^{P 301}$, and $\operatorname{trpl}^{302} \operatorname{InaD} \mathrm{D}^{P 215}$. The response latencies of both R1 and R2 are longer in $\operatorname{trpl}^{302}$ InaD ${ }^{P 215}$ than in trpl $^{302}$;rp ${ }^{P 301}$.


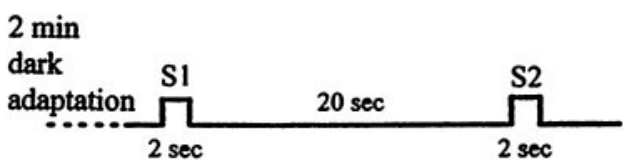

B

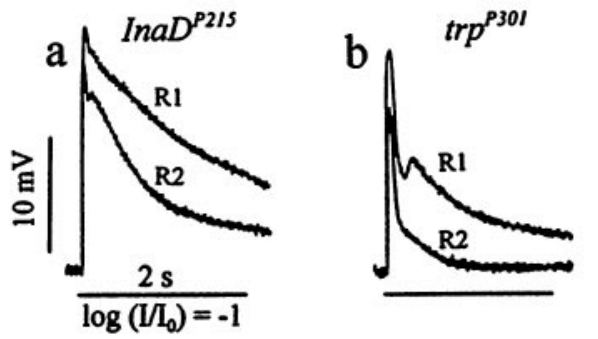

C
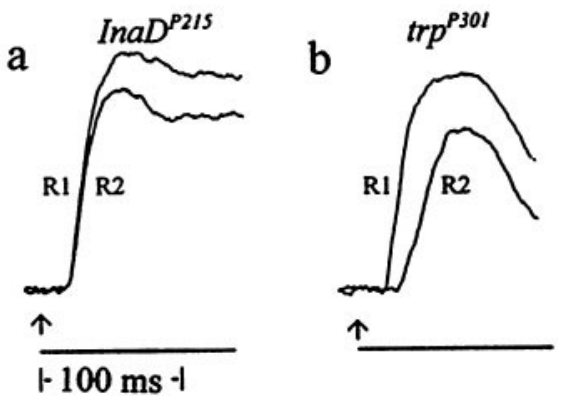

$2 \mathrm{~min}$

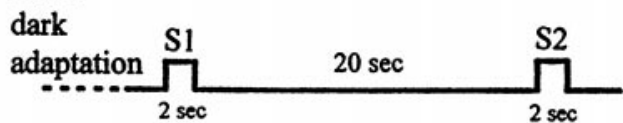

B a $\operatorname{trpl}^{302} ; \operatorname{trp}^{P 301} \operatorname{trpl}^{302} \operatorname{InaD} D^{p 215}$

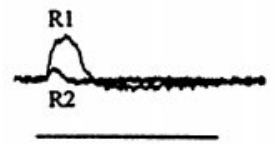

C

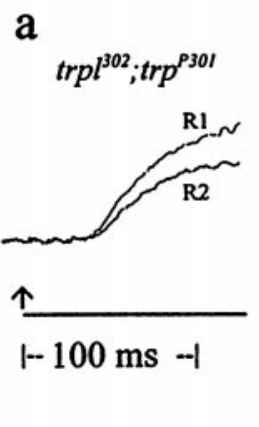

b $\operatorname{trpl}^{302} \operatorname{InaD} D^{p 215}$

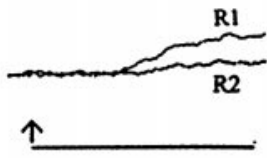

possible interpretation of these results is that a significant amount of TRP is associated, however weakly, with the signaling complex via interaction with the TRPL protein, thus contributing to the photoreceptor response, even when TRP cannot bind directly to the signaling complex itself. In the absence of TRPL, however, the association is lost and much of the response is lost also.
The idea that TRP and TRPL proteins interact need not necessarily be wedded to the above specific model. For example, the extent to which the TRP protein fails to bind to INAD in the intact photoreceptors of the InaD $D^{P 215}$ mutant is unclear because most of the binding studies were performed in vitro. Regardless of the extent of TRP binding to INAD in the InaD ${ }^{P 215}$ mutant, however, 
the results of this study suggest that the presence of TRPL stabilizes the TRP channel and allows the latter to contribute to the photoreceptor response in InaD $D^{P 215}$, implying physical interactions between these two proteins.

The molecular nature of interactions between TRP and TRPL proteins cannot be specified from the results of the present study. However, it is pertinent to note that previous workers showed interaction between TRP and TRPL in immunoprecipitation assays (Xu et al., 1997) and presented evidence for the formation of heteromultimeric channels when both TRP and TRPL are coexpressed in oocyte (Gillo et al., 1996) or cell culture (Xu et al., 1997) systems. Whatever the actual mechanism of interaction responsible for our results, the results suggest that, in addition to "functional interactions," direct "physical interactions" between TRP and TRPL also may be a part of the photoreceptor process.

\section{REFERENCES}

Bloomquist BB, Shortridge RD, Schneuwly S, Perdew M, Montell C, Steller H, Rubin G, Pak WL (1988) Isolation of a putative phospholipase C gene of Drosophila, norpA, and its role in phototransduction. Cell 54:723-733.

Chevesich J, Kreuz AJ, Montell C (1997) Requirement for the PDZ domain protein, INAD, for localization of the TRP store-operated channel to a signaling complex. Neuron 18:95-105.

Cosens DJ, Manning A (1969) Abnormal retinogram from a Drosophila mutant. Nature 224:285-287.

Gillo B, Chorna I, Cohen H, Cook B, Manistersky I, Chorev M, Arnon A Pollock JA, Selinger Z, Minke B (1996) Coexpression of Drosophila TRP and TRP-like proteins in Xenopus oocytes reconstitutes capacitative $\mathrm{Ca}^{2+}$ entry. Proc Natl Acad Sci USA 93:14146-14151.

Hamill OP, Marty A, Neher E, Sakmann B, Sigworth FJ (1981) Improved patch-clamp techniques for high-resolution current recording from cells and cell-free membrane patches. Pflügers Arch 391:85-100.

Hardie RC (1991) Whole-cell recordings of the light-induced current in dissociated Drosophila photoreceptor: evidence for feedback by calcium permeating the light-sensitive channels. Proc R Soc Lond [Biol] 245:203-210.

Hardie RC, Minke B (1992) The trp gene is essential for a light activated $\mathrm{Ca}^{2+}$ channel in Drosophila photoreceptors. Neuron 8:643-651.

Johnson EC, Pak WL (1986) Electrophysiological study of Drosophila rhodopsin mutants. J Gen Physiol 88:651-673.

Larrivee DC, Conrad SK, Stephenson RS, Pak WL (1981) Mutation that selectively affects rhodopsin concentration in the peripheral photoreceptors of Drosophila melanogaster. J Gen Physiol 78:521-545.
Minke B (1982) Light-induced reduction in excitation efficiency in the trp mutant of Drosophila. J Gen Physiol 79:361-385.

Minke B, Wu C-F, Pak WL (1975) Induction of photoreceptor voltage noise in the dark in Drosophila mutant. Nature 258:84-87.

Montell C (1999) Visual transduction in Drosophila. Annu Rev Cell Dev Biol 15:231-268.

Montell C, Rubin GM (1989) Molecular characterization of the Drosophila trp locus: a putative integral membrane protein required for phototransduction. Neuron 2:1313-1323.

Niemeyer BA, Suzuki E, Scott K, Jalink K, Zuker CS (1996) The Drosophila light-activated conductance is composed of the two channels TRP and TRPL. Cell 85:651-659.

Pak WL (1979) Study of photoreceptor function using Drosophila mutants. In: Neurogenetics genetic approaches to the nervous system (Breakfield X, ed), pp 67-99. New York: Elsevier-North Holland.

Phillips AM, Bull A, Kelly LE (1992) Identification of a Drosophila gene encoding a calmodulin-binding protein with homology to the trp phototransduction gene. Neuron 8:631-642.

Pollock JA, Assaf A, Peretz A, Nichols CD, Mojet MH, Hardie RC, Minke B (1995) TRP, a protein essential for inositide-mediated $\mathrm{Ca}^{2+}$ influx, is localized adjacent to the calcium stores in Drosophila photoreceptors. J Neurosci 15:3742-6370.

Reuss H, Mojet MH, Chyb S, Hardie RC (1997) In vivo analysis of the Drosophila light-sensitive channels, TRP and TRPL. Neuron 19: $1249-1259$.

Shieh B-H, Zhu MY (1996) Regulation of the TRP $\mathrm{Ca}^{2+}$ channel by INAD in Drosophila photoreceptors. Neuron 16:991-998.

Stühmer W, Conti F, Suzuki H, Wang X, Noda M, Yahagi N, Kubo H, Numa S (1989) Structural parts involved in activation and inactivation of the sodium channel. Nature 339:597-603.

Tsunoda S, Sierralta J, Sun Y, Bodner R, Suzuki E, Becker A, Socolich M, Zuker CS (1997) A multivalent PDZ-domain protein assembles signaling complexes in a G-protein-coupled cascade. Nature 388:243-249.

Tsunoda S, Sierralta J, Zuker CS (1999) Specificity in signaling pathways: assembly into multimolecular signaling complexes. Curr Opin Gen Dev 8:419-422.

Wong F, Schaefer EL, Roop BC, LaMendola JN, Johnston-Seaton D, Shao D (1989) Proper function of the Drosophila trp gene product during pupal development is important for normal visual transduction in the adult. Neuron 3:81-94.

Xu X-Z, Li H-S, Guggino WB, Montell C (1997) Coassembly of TRP and TRPL produces a distinct store-operated conductance. Cell 89: $1155-1164$.

Xu X-Z, Choudhury A, Li X, Montell C (1998) Coordination of an array of signaling proteins through homo- and heteromeric interactions between PDZ domains and target proteins. J Cell Biol 142:545-555. 